\title{
CONTORNOS DEL DEBIDO PROCESO EN EL DERECHO COLOMBIANO (II). TRES LECTURAS INTEGRADORAS EN TORNO A LA FAVORABILIDAD PENAL ${ }^{*}$
}

Outlines of Due Process in Colombian Law (ii). Three Readings around the Criminal Favorability*

\section{Miguel Antonio Morón Campos** Boris Julián Zetién Maciá}

Fecha de Recepción: Mayo 6 de 2013

Fecha de Aceptación: Mayo 13 de 2013

Sumario: 1. Introducción; Ubicación normativa de la Favorabilidad Penal en Colombia; 2. Aproximación Teórica: Las interpretaciones de la Favorabilidad Penal y los Tipos Penales en Blanco; 3. Recorrido y Matices Jurisprudenciales; 4. Referencias bibliográficas.

\footnotetext{
"Este trabajo corresponde a la Segunda parte del informe final de una investigación financiada por la Fundación Universitaria Colombo Internacional (Unicolombo), cuyo nombre es "La Constitucionalización del proceso penal en Colombia 2006-2010: Contornos, problemas y desafíos de la jurisprudencia Constitucional" desarrollada el interior del Grupo de Investigación Derecho en Contexto desde el semillero Estudios Punitivos. Fecha de Inicio de la investigación: Septiembre/2012; Fecha en que finalizó la investigación: Febrero 2013. derechoencontexto@unicolombo.edu.co

" Profesor de Criminología, Co-director del Grupo de Investigación Derecho en Contexto de la Fundación Universitaria Colombo InternacionalUNICOLOMBO.Email:mmoron@unicolombo.edu.co

"** Estudiante de IV semestre adscrito al grupo de investigación Derecho en Contexto desde la línea de Estudios Punitivos del programa de derecho de la fundación Universitaria Colombo Internacional (UNICOLOMBO) Cartagena. Email: boriszetienmacia@gmail.com
} 


\section{COMO CITAR ESTE ARTÍCULO (APA 6TH)}

Morón Campos, M. A., \& Zetién Maciá, B. J. (2013). Contornos del debido proceso en el derecho colombiano (II). Tres lecturas integradoras en torno a la Favorabilidad Penal. (Y. Carrillo De la rosa, Ed.) Revista Mario Alario D'filippo V(10), Págs. 88-97

\section{RESUMEN}

En las siguientes líneas se pretende hacer un análisis del principio de favorabilidad penal e identificar los matices con los que se proyecta este principio en el ordenamiento jurídico-penal colombiano. La tesis planteada busca responder a la siguiente pregunta: ¿Cuáles son los límites interpretativos que la jurisprudencia constitucional ha establecido para el principio de favorabilidad penal en relación con el debido proceso? Para resolverla, se abordan principalmente los diversos aspectos tópicos en los que la jurisprudencia constitucional ha sido recurrente, ofreciendo además una aproximación histórica y una ubicación normativa del mismo.

\section{PALABRAS CLAVE}

Debido proceso, Favorabilidad Penal, derecho penal, tipos penales, Lex Tertia.

\section{ABSTRAC}

In the following lines it is to make an analysis of the principle criminal favorability and identify the nuances with which this principle is projected in the Colombian criminal legal system. The thesis put forth seeks to answer the following questions: What are the limits that the constitutional interpretive case law has established for the principle criminal favorability regarding due process? To solve it, the various topical aspects that constitutional jurisprudence has been recurrent, also offering a historical approach and location of the same rules they are primarily addressed.

\section{KEYWORDS}

Due process, criminal favorability, criminal law, criminal types, Lex Tertia. 


\section{INTRODUCCIÓN: UBICACIÓN NORMATIVA DE LA FAVORABILIDAD PENAL EN COLOMBIA}

En el derecho colombiano el principio de favorabilidad tiene una amplia regulación tanto de carácter jurídico nacional y jurídico internacional, a través, de los tratados ratificados por el Estado. En este punto mostraremos las diferentes normas que consagran el principio de favorabilidad, convirtiéndolo así en pieza clave al momento de llevar a cabo un proceso penal, todo esto a causa de las diferentes normas nacionales e internacionales que lo consagran y protegen.

Como elemento integral del debido proceso en materia penal se encuentra consagrado en el Art. 29 de la Constitución del 91 que profiere lo siguiente; "En materia penal la ley permisiva o favorable, aun cuando sea posterior, se aplicará de preferencia a la restrictiva", el anterior artículo no es más que una réplica casi idéntica del Art. 26 consagrado en la constitución de 1886.

A su vez en la Ley 153 de 1887 el Artículo 44 consagraba el principio de favorabilidad, conteniendo la siguiente disposición:

Artículo 44. En materia penal la ley favorable o permisiva prefiere en los juicios a la odiosa o restrictiva, aun cuando aquella sea posterior al tiempo en que se cometió el delito.

Con Ley 599 del año 200 con la cual se reformó el Código Penal colombiano se siguió consagrando dicho principio el Artículo 6 de la citada ley, estipulando lo siguiente:

Artículo 6 "Nadie podrá ser juzgado sino conforme a las leyes preexistentes al acto que se le imputa, ante el juez o tribunal competente y con la observancia de la plenitud de las formas propias de cada juicio. La preexistencia de la norma también se aplica para el reenvío en materia de tipos penales en blanco.

La ley permisiva o favorable, aun cuando sea posterior se aplicará, sin excepción, de preferencia a la restrictiva o desfavorable. Ello también rige para los condenados. La analogía sólo se aplicará en materias permisivas".

De la misma manera el Código de Procedimiento Penal en su Artículo 6 de la Ley 906 del 2004 regula y contiene el principio de favorabilidad en las normas penales de carácter procesal, consagrando que:

Articulo 6 Legalidad. "Nadie podrá ser investigado ni juzgado sino conforme a la ley procesal vigente al momento de los hechos, con observancia de las formas propias de cada juicio.

La ley procesal de efectos sustanciales permisiva o favorable, aun cuando sea posterior a la actuación, se aplicará de preferencia a la restrictiva o desfavorable.

Las disposiciones de este código se aplicarán única y exclusivamente para la investigación y el juzgamiento de los delitos cometidos con posterioridad a su vigencia".

En regulación internacional el principio de favorabilidad también goza de una gran protección a 
través de los tratados que han sido ratificados por el Estado colombiano. En el Pacto Internacional de Derechos Civiles y Políticos, aprobado por la Ley 74 de 1968 establece lo siguiente:

Parte III. Artículo 15 “Nadie será condenado por actos u omisiones que en el momento de cometerse no fueran delictivos según el Derecho Nacional o Internacional. Tampoco se impondrá pena más grave que la aplicable en el momento de la comisión del delito. Si con posterioridad a la comisión del delito la Ley dispone la imposición de una pena más leve, el delincuente se beneficiará de ello.

2. Nada de lo dispuesto en este artículo se opondrá al juicio ni a la condena de una persona por actos u omisiones que, en el momento de cometerse, fueran delictivos según los principios generales del derecho reconocidos por la comunidad internacional".

Por su parte la convención Americana de Derechos Humanos, aprobada en nuestro país por la Ley 16 de 1972 consagra en principio de favorabilidad en su Artículo 9:

\begin{abstract}
“Nadie puede ser condenado por acciones u omisiones que en el momento de cometerse no fueran delictivos según el derecho aplicable. Tampoco se puede imponer pena más grave que la aplicable en el momento de la comisión del delito. Si con posterioridad a la comisión del delito la ley dispone la imposición de una pena más leve, el delincuente se beneficiará de ello".
\end{abstract}

Al conocer todo lo anterior es evidente la protección que tiene el principio de favorabilidad en nuestro ordenamiento jurídico por lo cual no es errado sostener que este constituye un elemento fundamental del debido proceso y que se hace necesaria su existencia en los estados sociales de derechos, qué se denominan democráticos, protectores de la constitución y los derechos fundamentales.

\title{
2. APROXIMACIÓN TEÓRICA. LAS INTERPRETACIONES DE LA FAVORABILIDAD PENAL Y LOS TIPOS PENALES EN BLANCO
}

El principio de Favorabilidad Penal en Colombia consagrado en la Constitución de 1991 venía siendo entendido según Carlos Suárez (2012) de forma ligada al paradigma "formalismo jurídico", el cual argumenta Diego López Medina ha sido dominante tradicionalmente en la cultura de nuestro país y en toda Latinoamérica. Claramente expone el profesor Medina, que, este paradigma puede ser entendido como una comprensión del derecho que niega la existencia al interior del mismo, de cualquier dimensión independiente de justicia o equidad, por lo cual el derecho es entendido como una actividad encaminada y dedicada al examen de los textos jurídicos preexistentes sin tener en cuenta las realidades, intereses o necesidades, pues se sostiene bajo el paradigma "formalista" que la interpretación del derecho arroja resultados definitivos y correctos, es decir, no existen vacíos en el sistema jurídico pues hay formas cognitivas para llenarlos. (Lopez, 2006)

En este punto del trabajo mostraremos como el paradigma "formalista" en relación con la concepción tradicional del Principio de Favorabilidad Penal, presenta una serie de inconvenientes o problemas jurídicos contemporáneos que ponen en crisis la mencionada noción tradicional. Para 
poder lograr lo anterior, se hace necesario comprender, en primer lugar, cuál es la concepción tradicional del principio de favorabilidad penal para luego así poder adentrarnos a conocer cuáles son esos problemas que acarrea dicha noción.

De la anterior explicación entorno al principio de favorabilidad penal puede entenderse lo siguiente:

1. El principio de favorabilidad se aplica únicamente sobre leyes en sentido formal, es decir, el Principio de Favorabilidad no puede emplearse en normatividades administrativas que en algunos casos complementan a la norma penal y mucho menos procede sobre jurisprudencias que interpreten o aclaren la norma.

2. El Principio de Favorabilidad supone aplicar cabalmente una de las leyes penales que se encuentran comprometidas en el tránsito legislativo, siendo imposible la posibilidad de que el juez pudiese tomar lo más beneficioso de una ley y otra, es decir, sin que se la posibilidad de construir una "Lex tertia" o tercera ley.

3. El Principio de Favorabilidad ha sido comprendido como una norma jurídica de todo o nada, que en toda ocasión debe emplearse plenamente, a lo cual no permite cumplimientos parciales ni ponderación con otras normas de carácter jurídico penal frente a las cuales pueda encontrarse en conflicto sobre un caso concreto.

El primer problema jurídico que se presenta frente a la concepción tradicional del principio de favorabilidad penal, es en torno a lo se conoce en el Derecho Penal como Tipos o Normas Penales en Blanco, las cuales son entendidas como "aquellas normas que determinan la sanción aplicable, describiendo solo parcialmente el tipo delictivo correspondiente y confiando la determinación de la conducta punible o su resultado a otra norma jurídica a la cual reenvía expresa o tácitamente" (Cury, 1988). En otras palabras los tipos penales en blanco son normas construidas a partir de una técnica legislativa de reenvió, que surge a causa de la complejidad y volatilidad de algunas materias de regulación, por lo cual el legislador remite al intérprete a otra u otras disposiciones para disponer en qué consiste la conducta punible.

Los tipos penales en blanco han sido clasificadas de dos formas: tipos penales en blanco propios y tipos penales en planco impropios.

La Ley Penal en Blanco es propia, cuando confía la complementación del precepto a una instancia legislativa de inferior jerarquía (disposición reglamentaria, acto administrativo, orden de policía, etcétera); en cambio es impropia cuando remite a otra ley emanada de la misma instancia legislativa (ley formal). Las Leyes en Blanco, a su o impropias, a su vez, pueden subdistinguirse en aquellas que hacen un reenvío interno, es decir, que remiten a otra de sus propios artículos, y las que realizan un reenvío externo, esto es, remiten a otra ley formal. (Cury, 1988)

La problemática que surge es entorno a que si es plausible la aplicación del Principio de Favorabilidad frente a las Normas Penales en Blanco "propias" al momento que la norma de reenvío administrativa sufra un cambio ulterior favorable al procesado o condenado. 
Basándonos en la concepción tradicional del Principio de Favorabilidad claramente es imposible que este se aplique en los casos de Normas Penales en Blanco que remiten a normatividades administrativas, pues como sabemos la concepción tradicional supone que este solo es aplicable tratándose de un tránsito de leyes en sentido estricto o formal. A continuación nos dedicaremos analizar cuál ha sido la postura de la Jurisprudencia Colombiana en torno a esta problemática.

En el trabajo citado de Suarez se sostiene que en 1980 la Corte Suprema de Justicia en Sala de Casación Penal (Corte Suprema de Justicia-Sala Penal. Sentencia 15/feb/1980 (Corte Suprema de Justicia. Sala de Casación Penal, 1980) siguiendo las tesis del penalista alemán Edmund Mezger del "total estado jurídico en el que descansa la punibilidad" reconoció que el principio de favorabilidad también se predicaba sobre esta clase de normas penales en escenario de sucesión de normas de reenvió de tipo infra-legal, colocando como único límite la cosa juzgada, es decir, que antes de dictarse la sentencia definitoria haya surgido la modificación extra penal del tipo o de sus principales componentes.

Los argumentos de la corte utilizados en ese momento fueron algunos como; que por "ley penal" no debe entenderse tan solo el conjunto de normas establecidas en el Código Penal sino todas las disposiciones restantes del ordenamiento jurídico que tenga correlación de manera directa con la primera.

Otro argumento empleado por la jurisprudencia en la misma sentencia es que tanto la norma objetiva de derecho (se refiere al juicio de valoración sobre determinadas conductas) y la ley penal en sentido estricto (estados para determinar el objeto de la protección como la norma protectora) hacen parte del "estado jurídico en el que descansa la punibilidad". Teniendo en cuenta lo anterior la corte sostiene que se crea una atenuación en el estado jurídico, no simplemente cuando se modifican favorablemente al reo las disposiciones que pertenecen a esta última (ley penal en sentido estricto) sino que a su vez cuando sufren variaciones las primeras (norma objetiva de derecho), pues esto implica que el legislador ya no considera que debe castigarse determinada violación del orden normativo cultural o que la sanción deber ser menor por haber mermado la gravedad de la lesión a dicho ordenamiento.

En otra sentencia del año 2007 se sostiene que la Corte Suprema de Justicia en su Sala de Casación Penal acoge la tesis tradicional que considera la no aplicabilidad del Principio de Favorabilidad cuando se trate de normas de reenvió de naturaleza infra-legal en origen de tipo penales en blanco. (Gomez \& Suarez, 2012)

La Corte argumenta para sostener lo anterior que en los denominados "Tipos Penales en Blanco" en primer lugar es aceptado que la ley posterior puede afectar (Corte Suprema de Justicia-Sala de Casación Penal. R. 27-337/2007) (Corte Suprema de Justicia - Sala Penal, 2007) no solo los textos de la propia norma penal, sino que de igual forma las disposiciones extrapenales que inciden indirectamente en la extensión del tipo, ya que esa normatividad se integra al tipo penal y constituye con él una unidad inseparable. No obstante la jurisprudencia aclara lo anterior argumentando que si la transformación favorable del tipo es provocada por una reforma de la propia norma en blanco ella debe favorecer sin limitaciones a los autores de hechos llevados a cabo 
con antelación a su vigencia y en el segundo caso, la Ley y la dinámica de la vida real plantea una serie de dificultades, que para poder solucionar se hace necesario un análisis profundo percibir algunas distinciones.

La Corte Suprema entiende el uso de Leyes Penales en Blanco como una técnica legislativa cuyo fin es proteger relaciones sociales, que necesitan ser actualizadas constantemente. En ese sentido es claro que en la mayoría de los eventos la modificación de la norma integradora no es una revaluación de la conducta punible, puesto que en las circunstancias en que su momento fue ejecutada, continua siendo inaceptable y atenta contra el bien jurídico protegido de manera permanente por el Tipo en Blanco.

El comportamiento llevado a cabo y que adquiere connotaciones de punible, es construido en un dato histórico relevante y fijo, podría ser sometido a valoración mas no de cambio (Gómez \& Suárez, 2010), mientras no sea el legislador quien introduzca normas que eliminen la valoración de calidad nociva del conducta punible y mientas eso no suceda la conducta será vista con la misma dimensión en los diferentes tiempos que se analice.

\section{RECORRIDO Y MATICES JURISPRUDENCIALES}

Para finalizar, analizaremos la controversia que ha venido surgiendo a través de los años, la cual trata sobre si es posible la aplicación del principio de favorabilidad a las líneas jurisprudenciales de las altas Cortes en Colombia.

Es sabido que la Corte Constitucional hace años viene sosteniendo la obligatoriedad de sus precedentes jurisprudenciales, tanto, en sus fallos de constitucionalidad y de tutela, sometiendo a lo dictado tanto a ciudadanos como a jueces. Sentencias como la C-113 del 93 nos enseña que fallos de constitucionalidad abstracta son de obligatorio cumplimiento (erga omnes), además aquellos aportes de las motivaciones o consideraciones de la corte "que guarden una unidad de sentido con el dispositivo de la sentencia" (Corte Constitucional de Colombia. Sentencia C 113 de 1993 () La Corte Constitucional de igual forma reconoció los efectos vinculantes (erga omnes) a los fallos de las otras altas Cortes en Colombia. En la sentencia C-836 de 2001 en la cual se analizó en Artículo 4 de la Ley 169 de 1896 sobre la "doctrina probable", la corte fue enfática al sostener que la jurisprudencia del Consejo de estado y de la Corte Suprema de Justicia tiene efectos vinculantes para todos los jueces del estado().

Entendiendo lo anterior es claro el valor normativo e importante que ha adquirido a través de los años las decisiones de las altas cortes en nuestro país, pero al momento de que estas decisiones se utilicen de manera retroactiva o ultractiva atendiendo al principio de favorabilidad, la posición de las altas cortes en principio ha sido tajante al negar dicha posibilidad. En la sentencia del 28 de noviembre de 2002 la Corte Suprema de Justicia argumentando su decisión bajo el paradigma del "formalismo jurídico" y entendiendo el Principio de Favorabilidad de una forma Alexyana, es decir, aplicación de todo o nada sostuvo; "el principio de favorabilidad implica como referente tener la ley, y no un criterio que apenas es disciplina auxiliar de la actividad". 
Por otro lado, al momento del estudio del principio de favorabilidad se asume implícitamente como la posibilidad del surgimiento de una ley tercera, es decir, poder tomar y aplicar preceptos favorables al indiciado de una y otra ley que se sucedan en el tiempo. Tradicionalmente la doctrina desecha la anterior posibilidad argumentando que la aplicación de una y otra ley en un escenario de tránsito legislativo convertiría al juez en un legislador, ya que construiría una tercera ley y además violaría el principio de la tridivisión del poder público, así mismo la posición jurisprudencial no difería de la concepción doctrinal; de rechazar la posibilidad de combinar los preceptos que más sean favorables al indiciado de varias leyes en un escenario de sucesión legislativa.

Un ejemplo claro es la Corte Suprema de Justicia en sentencia del 24 de abril de 1981 (Corte Suprema de Justicia. Sala Constitucional, 1981), sostuvo:

Esa aplicación debe ser integral, esto es, en todo lo que se relaciona con el evento jurídico que se está resolviendo, sin que sea permitido tomar de cada una de las normas en comparación lo que favorece y desechar lo que perjudica, pues ello equivaldría a crear una especie de tercera norma, especial para el caso.

A su vez en pronunciamientos más recientes la corte empezó a replantear su postura tradicional en torno a la "Lex tertia". Suárez sostiene, que después de la entrada en vigencia del Código Penal (Ley 599 del 2000) la jurisprudencia penal ha contemplado de forma positiva la posibilidad de combinar, atendiendo al principio de favorabilidad, preceptos de las leyes que se suceden en el tiempo a través de una nueva concepción de la expresión "Lex tertia".

Sentencias como la del 3 de septiembre de 2001 en sala de casación penal de la Corte Suprema de Justicia, marcaron un hito importante en cuanto al replanteamiento en torno a la "Lex tertia", que se seguiría repitiendo por parte de la Corte Suprema en sentencias posteriores; un ejemplo de ello la sentencia del 6 de octubre de 2004 que expone enfáticamente lo siguiente:

El fenómeno conocido con el nombre de conjugación, conjunción o combinación de disposiciones, igualmente llamado Lex tertia, tiene cabal cabida en nuestro medio. Por tanto, frene a la sucesión de leyes en el tiempo es perfectamente posible tomar de una forma posible tomar de una norma lo favorable y desechar lo odioso, así como tomar de la otra u otras lo benigno y dejar de lado lo desfavorable.

En distintos espacios académicos se hace común la discusión en torno a la aplicabilidad del Principio de Favorabilidad sobre normas procesales penales, la primera tesis en torno a la aplicación o no del principio frente a normas procesales penales sostienen que este principio solamente es aplicable de normas penales sustanciales o materiales, por lo cual argumentan que a diferencia al momento de aplicación del principio en normas procesales está en que, estas últimas, solo se refieren a establecer trámites procesales, meramente instrumentales sin valor alguno y político-criminalmente neutrales lo cual hace imposible sostener que algunos sean más favorables que otros.

La anterior tesis cae por su propio peso y carece de toda validez pues como sostiene Carlos Suárez a la luz de la Constitución en su Artículo 29 este no distingue de normas sustanciales o procesales al 
momento de consagrar en ella el principio de favorabilidad. De igual forma sostener el argumento de que las normas procesales contienen un carácter avalorado, políticamente neutro es desmitificado porque estas normas procesales afectan de igual o quizás en mayor forma que las normas penales sustanciales a los Derechos Fundamentales, es misma postura es compartida por la Corte Constitucional en la en la sentencia C-207 del once de marzo del 2003:

Las leyes de procedimiento no solo están destinadas para fijar competencias y disponer ritualidades adjetivas de los juicios criminales. Muchas de sus disposiciones, las más importantes, consagran los recursos contra las providencias judiciales, los términos probatorios, el debate dentro del plenario, la asistencia profesional del acusado, los medios de defensa, los recursos extraordinarios, los motivos de detención preventiva, entre otras cosas, todas ellas fundamentales, que de un procedimiento a otro puedan, de modo esencial, afectar los derechos del sujeto pasivo de la acción penal. (Corte Constitucional de Colombia, 2003d)

Se hace entendible porque el legislador en la Ley 906 de 2004 (Art. 6, inciso 2) establece que: "la ley procesal de efectos sustanciales permisiva o favorable, aun cuando sea posterior a la actuación, se aplicará de preferencia a la restrictiva o desfavorable", lo que el legislador intentó no fue más que posibilitar la aplicabilidad del Principio de Favorabilidad de la ley procesal penal, por lo cual se puede sostener que el Principio de Favorabilidad opera con total extensión tanto en leyes sustanciales y procesales, un ejemplo claro es la sentencia C-592 del nueve de junio del 2005 :

Sobre este punto debe la Corte señalar que tratándose de la aplicación del principio de favorabilidad en materia penal, no cabe hacer distinción entre normas sustantivas y normas procesales, pues el texto constitucional no establece diferencia alguna que permita un trato diferente para las normas procesales. (2005)

\section{REFERENCIAS BIBLIOGRÁFICAS}

CORTE SUPREMA DE JUSTICIA. Sala de Casación Penal. República de Colombia 15 de febrero de 1980.CORTE SUPREMA DE JUSTICIA. República de Colombia 24 de abril de 1981.

Demanda de inconstitucionalidad contra una parte del inciso segundo del Artículo 21 del Decreto 2067 de 1991, C-113/1993 (Corte Constitucional de Colombia 25 de marzo de 1993).

Acción pública de inconstitucionalidad en la que se demanda la inexequibilidad del Artículo 40 de la Ley 169 de 1896., C-836/2001 (Corte Constitucional de Colombia 9 de agosto de 2001).

Acción pública de inconstitucionalidad que demanda la inexequibilidad del Artículo 17 de la Ley 144 de 1994., C-207/2003 (Corte Constitucional de Colombia 11 de marzo de 2003d).

Demanda de inconstitucionalidad contra las expresiones "Las disposiciones de este código se aplicarán única y exclusivamente para la investigación y el juzgamiento de los delitos cometidos con posterioridad a su vigencia" contenida en el inciso final del, C-592/2005 (Corte Constitucional de Colombia 9 de junio de 2005). 
RADICADO N-27 337 (Corte Suprema de Justicia Sala Penal. República de Colombia 23 de agosto de 2007).

CURY, E. (1988) "La Ley Penal en Blanco". Bogotá: Temis.

GÓMEZ, C., \& SUÁREZ, C. (2012) "Estudios de Derecho Penal I". Bogotá: Universidad Jorge Tadeo Lozano.

LÓPEZ, D. (2006) "El Derecho de los Jueces". Bogotá: Legis. 